\title{
A Rare Case of Bilateral Inflammatory Linear Verrucous Epidermal Nevus
}

\section{Yousuf Abd Mallick', Nausheen Yaqoob²}

1-2. Dermatology Unit 1 Pathology Unit, ${ }^{2}$ The Indus Hospital Karachi, Pakistan

Correspondence to: Dr. Yousuf Abd Mallick, Email: dryousuf2006@yahoo.com, ORCiD:0000-0002-8250-3319

\begin{abstract}
Inflammatory linear verrucous epidermal nevus (ILVEN) is rare, mosaicism-induced linear inflammatory dermatoses with early childhood presentation and persistence for whole life in majority of cases. No definite gene or genetic locus has been nominated yet. Treatment is mostly aimed for relief of symptoms and for cosmetic concerns.Here, authors are reporting a case of 6-year-old girl presented with history of extremely pruritic, erythematous, linear plaques following the lines of Blaschko over all 4 limbs, trunk and flexures since infancy. Clinical impression of bilateral ILVEN was confirmed by histopathology. No systemic abnormality was detected. Bilateral ILVEN is extremely rare entity and it should be documented whenever encounter.

Keywords: Inflammatory linear verrucous epidermal nevus, histopathology, mosaicism.
\end{abstract}

This is an Open Access article distributed under the terms of the Creative Commons Attribution Non-Commercial License (http:// creative commons. org/licenses/by-nc/4.0) which permits unrestricted non-commercial use, distribution, and reproduction in any medium, provided the original work is properly cited.

\section{INTRODUCTION}

Inflammatory linear verrucous epidermal nevus (ILVEN) is a seldomly encountered ailment and believed to be caused by an unknown postzygotic mutation, which is rescued by genetic mosaicism. ${ }^{1}$ ILVEN presents as intensely pruritic, erythematous, psoriasiform or lichenified verrucous papules coalesce to form linear plaques following the lines of Blaschko. Majority of cases presented before 5 years of age. ${ }^{2}$ Females are affected four times more with striking unilateral lower limb involvement. Familial ILVEN is also a well establish entity but gene identification has yet to be done. In the literature, few adult-onset cases have also been reported. ${ }^{2}$ Bilateral or generalized presentation is very rare and needs documentation.

\section{CASE REPORT}

A 6-year-old female child, from a consanguineous marriage, presented to the Dermatology department of the Indus Hospital, Karachi with more than 5-year history of extremely pruritic linear and semi-circular eruptions over different parts of her body. According to father, lesions first appeared during infancy on left side of her neck, then gradually spread to involve other parts. At the time of presentation; her neck, all four limbs, trunk and many flexures of the body were affected. The progression of lesions stopped one year back. There was no history of erythroderma, collodion at birth, blistering or photosensitivity. Family history for genetic skin disorder was negative. The condition slightly improved in summer and aggravated in winter season. The child was apparently healthy with good schooling records and achieved all milestones at appropriate ages. Systemic review was unremarkable. Other siblings were healthy and had no signs of skin disease. Examination revealed multiple, bilaterally symmetrical, erythematous, scaly, eroded, linear plaques involving her neck, axillae, all four extremities, trunk, groins and popliteal fossae, tracing the lines of Blaschko (Figures 1-3). Left side was more involved as compared to right. Two fingernails and 1 toe nail were also affected. Many scratch marks, erosions and bleeding points were obvious. Face, genitals, palms and soles were spared. No involvement of any mucosa. Scalp showed normal hair growth and was devoid of lesions. Vision and hearing were intact. Gait and range of movement at all joints were normal. There was no length discrepancy and no gross abnormality was detected in any of the limbs. Various cutaneous side effects of misuse of topical steroids were evident at many places like striae, hypopigmentation, hypertrichosis and atrophy of unaffected skin. Systemic examination revealed no abnormality in the child.

Histopathological examination showed hyperkeratosis, acanthosis and papillomatosis with classical alternating bands of parakeratosis and orthohyperkeratosis. Granular layer was absent below patches of parakeratosis, while thickening of granular layer (hypergranulosis) beneath the dense orthokeratosis was obvious. Rete ridges were elongated and thickened. Collection of neutrophils in stratum corneum and mild perivascular lymphocytic infiltrate in dermis were present (Figure 4).

Based on these characteristic clinical features followed 
by histopathological confirmation, a diagnosis of bilateral ILVEN was made by authors. Absence of skeletal, neurological and ophthalmic abnormalities helped us to exclude Epidermal Naevus Syndrome (ENS). Emollients, antihistamines and follow up were advised. Patient was referred for Carbon dioxide $\left(\mathrm{CO}_{2}\right)$ ablative Laser treatment to another specialized center.
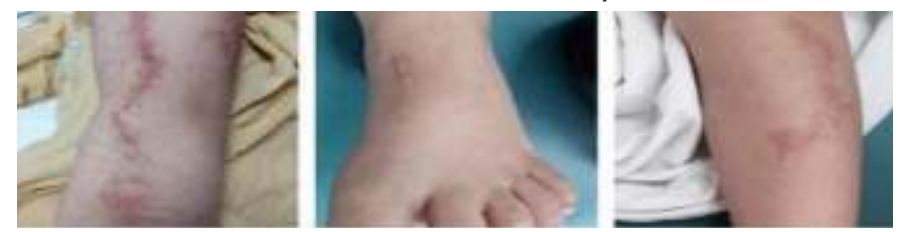

Figure 1: ILVEN involving Left arm, fore-arm, foot and leg
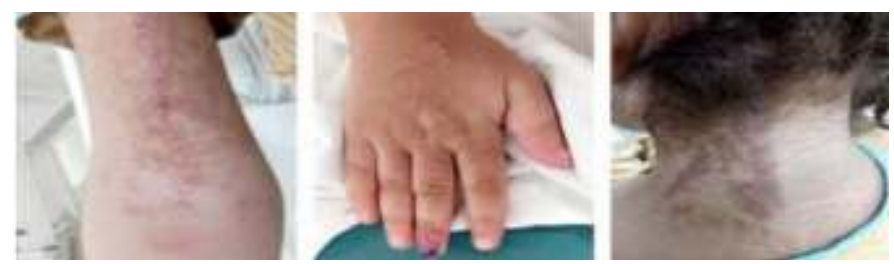

Figure 2: ILVEN involving Right arm, elbow, hand and neck
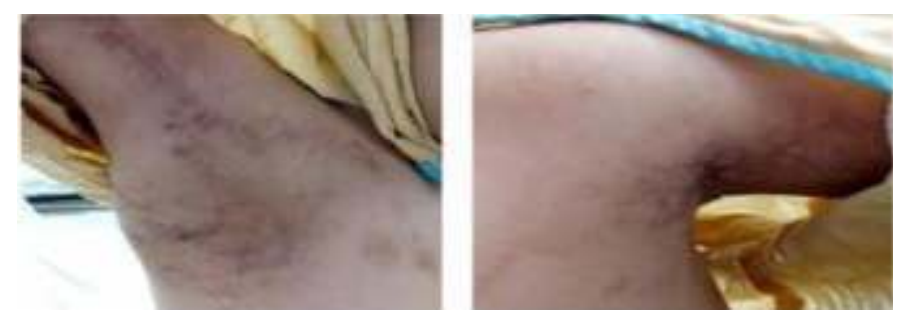

Figure 3: ILVEN involving Right and Left axillae

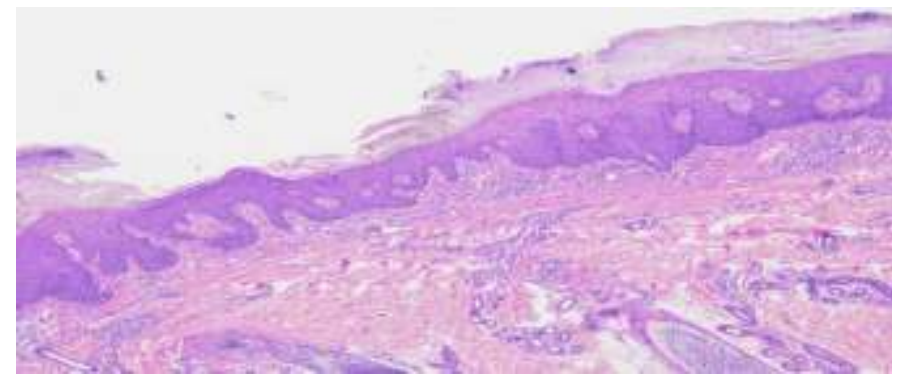

Figure 4: Histology showing well distinguished alternate bands of orthokeratosis with hypergranulosis and parakeratosis with agranulosis ( $\mathrm{H}$ and E, $\times 20$ )

\section{DISCUSSION}

The terminology of "Inflammatory linear verrucous epidermal nevus" was first proposed by a German Dermatologist Paul G. Unna in $1896 .^{1}$ He labeled it as congenital malformation or hamartoma derived from embryonic ectoderm. Later on, Kaidbey and Kurban in 1971, described ILVEN as a separate entity. Altman and Mehregan, in 1971, studied 25 cases of ILVEN and proposed the first ever diagnostic criteria for this disease. ${ }^{3}$ This criteria is still widely in use and consists of 6 points which are: early age of onset $(75 \%$ of cases with onset before 5 years of age, and 50\% before 6 months), female predominance ( $M: F$ ratio 1:4), marked pruritus, involvement of the left leg in a great majority, resistance to therapy, and a peculiar psoriasiform and inflammatory histopathology.

For unknown reasons; ILVEN usually affects females and involves single lower extremity with left sided preference. ${ }^{2}$ It can be congenital or adult-onset, but majority develops this disease in early phase of childhood. Family history is usually absent. Presentation is classical with intensely pruritic, erythematous, psoriasiform, scaly, linearly arranged coalescing papules and plaques of variable lengths. Following the Blaschko lines is a characteristic feature. ${ }^{4}$ The lesions tend to persist for many years despite of treatment and may become verrucous and lichenified.

Due to its clinical and histopathological resemblance to psoriasis, many early cases were believed to be labelled as linear psoriasis in the literature. ${ }^{5}$ So, Dupre and Christol, in 1977, proposed the histopathological criteria for diagnosis of ILVEN in order to enhance its detection and accuracy of reporting. ${ }^{6}$ Dupre and Christol's histopathological criteria consists of two components. Both should be present to make a diagnosis.

1) Psoriasiform epidermal hyperplasia with classical elongation of rete ridges \& thinning of suprapapillary epidermis; parakeratosis, tortuous dermal capillaries and lymphocytic infiltration in papillary dermis.

2) Sharply separated alternate bands of orthokeratosis with hypergranulosis, and parakeratosis with hypo or agranulosis.

Differentials of ILVEN include all those disorders which have linear or naevoid variants (like psoriasis, Darier's disease, porokeratosis, lichen planus, lichen simplex chronicus) or those which usually present in a linear pattern (like adult blaschkitis, lichen striatus, porokeratotic eccrine ostial dermal duct nevus (PEODDN)). Syndromes to be distinguished from ILVEN are Epidermal Naevus Syndrome (ENS) and congenital hemidysplasia with ichthyosiform nevus and limb defects syndrome (CHILD syndrome)..$^{12}$

These differentials can be easily ruled out by proper history, detailed clinical examination and characteristic histopathological features of ILVEN except linear psoriasis. To exclude linear psoriasis; besides clinical and histopathological criteria, immunohistochemical (IHC) staining pattern must also be used wherever available. There is reduced number of Ki-67 positive nuclei in epidermis, focal pattern of anti-keratin 16 and diffuse pattern of anti-keratin 10 in ILVEN. The reverse is present in cases of linear psoriasis. ${ }^{4} \mathrm{IHC}$ staining of epidermal keratinocytes is positive for IL-36 $\gamma$ in psoriasis, while its negative in ILVEN. ${ }^{7}$ CHILD syndrome, 
an 'X linked dominant' condition, can be easily differentiated from ILVEN due to presence of congenital hemidysplasia and limb defects. The nevus is present since birth, completely asymptomatic and covered with waxy scales. When epidermal nevus is associated with underlying systemic anomalies, then it is termed as 'ENS'. Most common structural and functional abnormalities seen in ENS are present in neurological, ophthalmological and skeletal systems. This condition is also sporadic and degree of pruritus is similar to ILVEN. Although, ILVEN is unilateral, but few reports of bilateral presentation have been published. The first ever case report dates back in 1977 by Dupre and Christol from France. ${ }^{8}$ Their patient had bilateral lower limb and nails involvement. Later on, a very similar case was notified from The Netherlands in 1983 by Landwehr and Starink. ${ }^{9}$ None of them reported any associated internal disorder, neither morphological abnormality of limbs.

In 1978, Cheesbrough and Kilby, delineated a case of ILVEN in 32-year-old woman with extensive bilateral lower limbs, flexures, trunk and face involvement but no systemic involvement was identified in her. ${ }^{5}$ She developed lesions first time during pregnancy. Although exacerbations reported in subsequent pregnancies but no adverse obstetrical outcome was reported.

Oram and colleagues ${ }^{10}$ reported bilateral ILVEN in an 8year-old boy who also had psoriasis without any family history. Both diseases were biopsy proven. Yaghoobi et al. from Iran, ${ }^{11}$ also reported bilateral ILVEN in a 2-yearold girl. Mishra and colleagues described a 3-year-old boy with generalized, bilaterally symmetrical plaques of ILVEN following lines of Blaschko. All four extremities, flexures, trunk and genitals were grossly involved, and that case showed additional feature of epidermolysis on histology besides ILVEN pathtology. ${ }^{12}$ All these kids were healthy and had no physical, developmental or neurological abnormality at the time of presentation. Our case is similar to all above-mentioned cases with extensive bilateral involvement but absence of systemic features. Our case fulfils all clinical and histopathological criteria for diagnosis of ILVEN.

\section{CONCLUSION}

ILVEN is uncommon and bilateral presentation of ILVEN is extremely uncommon. Very few cases published in the literature. Occasionally, ILVEN cases have been reported with systemic anomalies. These cases should be differentiated from ENS and CHILD syndromes.

Authors believe that proper identification and reporting of cases of ILVEN along with genetic studies, whether unilateral or bilateral, associated with anomalies or not, will help in proper classification of all these cases in future.

Received: May 05, 2020

Accepted: August 20, 2020

\section{REFERENCES}

1. Tiwary AK, Mishra DK. A unique porokeratotic variant of inflammatory linear verrucous epidermal nevus. Indian J Paediatr Dermatol 2017;18:237-40.doi: 10.4103/23197250.20608.

2. Tiwary AK. Adult onset of inflammatory linear verru-cous epidermal nevus: truly a rare experience. J Pak Assoc Dermatol2019;29:253-6.

3. Altman J, Mehregan AH. Inflammatory linear verrucose epidermal nevus. Arch Dermatol 1971;104:385-9.

4. Kaushal A, Gahalaut P, Mishra N, Rastogi MK. Generali-zed ILVEN or blaschkoid psoriasis: a persistent dilemma. Nepal J Dermatol VenereolLeprol 2019;17:79-82.doi: 10.3126/njdvl.v17i1.22048.

5. Cheesbrough M J, Kilby $P$ E. The inflammatory linear verrucous epidermal naevus: a case report. Clin Exp Dermatol 1978;3:293-8.

6. Dupre A, Christol B. Inflammatory linear verrucose epidermal nevus. a pathologic study. Arch Dermatol 1977;113:767-9.

7. Tanita K, FujimuraT, Sato Y, Lyu C, Aiba S. Widely spread unilateral inflammatory linear verrucous epidermal nevus(ILVEN). Case Rep Dermatol 2018;10:170-5.doi: $10.1159 / 000489876$.

8. Dupre A, Christol B. Bilateral inflammatory linear verrucous epidermal nevus localized on the lip and with minimal histological lesions. Ann Dermatol Venreol. 1977;104:163-4.

9. Landwehr AJ, Starink TM. Inflammatory linear verrucous epidermal naevus. Report of a case with bilateral distribution and nail involvement. Dermatologica 1983;166:107.

10. Orm Y, Arisoy AE, Hazenci E, Gurer I, Mueezinoglu B, Arisoy Es. Bilateral inflammatory linear verrucous epidermal nevus associated with psoriasis. Cutis 1996; 57:275-8.

10. Orma, Arisoy A E, Hazneci E, Gurerl, Muezzinoglu B, Arisoy ES. Bilateralinflammatory linear verrucous epidermal nevus associated with psoriasis. Cutis 1996;57:275-8.

11. Yaghoobi R, Sina N, Ranjbari N, Rafiei R. Bilateral inflammatory linear verrucous epidermal nevus. Iran J Dermatol 2006;9:19-23.

12. Mishra V, Saha A, Bandyopadhyay D, Das A. Bilateral systematized epidermolytic verrucous epidermal nevus: a rare entity. Indian J Dermatol 2015;60:397-9. doi: 10.4103/0019-5154.160495 